\title{
Tiempo no productivo en pozos de dos secciones, caso de estudio Ecuador
}

\author{
Marco Loaiza, Diego Ayala, Henry Torres, Silvia Ayala * \\ Departamento de Ciencia de la Energía y Mecánica. Universidad de la Fuerzas Armadas ESPE. \\ Código Postal: EC050102. Latacunga. Ecuador. \\ E-mail: saayalat@yahoo.com
}

\begin{abstract}
Resumen
Con los costos actuales de los servicios de perforación y un mercado totalmente inestable que ha ubicado al precio del barril de petróleo en sus niveles más bajos en la última década; resulta imperativo minimizar cualquier evento que retrase el avance de perforación de un pozo. La propuesta de implementar una herramienta de planificación desarrollada a partir de los resultados de la investigación brindará a ingenieros y operadores un recurso que contribuirá a terminar un pozo dentro de los plazos establecidos con apego al presupuesto, culminando de manera exitosa cada pozo, con campañas de perforación cada vez más cortas y con un retorno sobre la inversión más alto al poner a producir los pozos en menor tiempo. Como resultado del trabajo se expone el impacto económico de los eventos no planificados.
\end{abstract}

Palabras clave: Tiempo no productivo, Problemas al perforar, Optimización de la perforación.

\section{Non-productive time in tow-section well construction case study in Ecuador}

\begin{abstract}
Considering the current costs for drilling services within a market being completely unstable that have placed the oil barrel at lowest prices in the last decade, it is extremely important to reduce every activity that delay the normal course of well drilling. The proposal for a resource of planning which is obtained from the present research. This resource was developed for engineers and operators aiming at ending of wellbore within scheduled time with the stablished budget, in this way being the operation successful in every well into more quick tasks with higher return on investment by starting early the well production. The economic impact of unplanned events is exposed as a result from this paper.
\end{abstract}

Keywords: Non-productive time, Drilling problems, Drilling optimization.

\section{Introducción}

Durante varios años la industria petrolera se ha enfrentado a problemas asociados con pega de tubería y la pérdida de circulación, siendo la pega diferencial uno de los inconvenientes que mayor impacto ha tenido en el costo final del pozo. (Adams, 1977) (Wisnie \& Zhiwei, 1994). Típicamente el NPT (Non Productive Time, en español Tiempo no productivo) representa hasta el 32 por ciento de los costos en los eventos de perforación, algunos de los eventos no planeados llegan a ser tan severos que pueden paralizar el programa de perforación (Halliburton, 2013). Los problemas de inestabilidad del pozo (Portilla, Suárez, \& Corzo, 2012) en operaciones de exploración y perforación cuestan a la industria de la perforación más de 100 millones de dólares por mes y en todo el mundo posiblemente tanto como mil millones de dólares anuales (Moazzeni, Nabaei, \& Azari, 2011).

Las grandes empresas operadoras y prestadoras de servicio, alguna vez han invertido sus recursos y su tecnología para poder reducir una medida que se ha instaurado como un parámetro de calidad en la perforación de pozos de petróleo, gas y geotermia, el llamado NPT (Weakley, 1990).

Cita: Loaiza, M., Ayala, D., Torres, H. y Ayala, S. (2018). Tiempo no productivo en pozos de dos secciones, caso de estudio Ecuador. Revista Fuentes: El reventón energético, 16(1), 7-17. 
El presente estudio tiene como objetivo ayudar a mejorar los programas de perforación, para tener un impacto positivo en la reducción de eventos no planificados y minimizar los riesgos al perforar.

\section{Definición de NPT}

No existe una sola definición de NPT, varios autores y empresas describen un concepto que se adapta a sus necesidades, aquí se citan algunos; el NPT se define como el tiempo que la operación de perforación ha cesado o la tasa de penetración es muy baja. El tiempo dedicado a la pesca, la pega de tubería, el transporte de herramientas, clima, la pérdida de circulación y viajes no programados dentro y fuera del pozo pueden contarse como NPT. (Nabai, Alireza, \& Moradinejad, 2016). Además, se describe al NPT como el tiempo en el que las operaciones de perforación no se producen, es decir, al extraer del fondo del agujero la sarta de perforación (Ayala, Benítez \& Valencia, 2017) y reanudar las operaciones a la misma profundidad (Alappat, 2015). También se detalla al NPT como el período de tiempo de eventos o actividades en las operaciones, que retardan el avance de las actividades de construcción y/o rehabilitación de un pozo (Ayala, Gómez \& León, 2011) según lo planificado. Se inicia desde que se evidencia una actividad no productiva, hasta que se encuentran de nuevo las condiciones operacionales productivas que tenía antes del evento improvisto (Morán, 2014) (Zapata, 2011).

\section{Operaciones productivas}

Son los pasos y tareas operativas programadas, de las cuales deriva el registro del tiempo real al perforar
(Bonilla \& Buestán, 2013). En la tesis Modelo de Control de Costos de Perforación de Pozos para los Campos Cuyabeno-VHR de Petroamazonas se propone la clasificación de las operaciones productivas. (Molina, 2014), por lo tanto, cualquier otra actividad deberá ser considerada como una tarea no productiva, lo cual nos permite definir la clasificación de las tareas no productivas.

\section{Operaciones no productivas}

Se define como una operación o tarea innecesaria, no contempladas dentro del planeamiento del pozo (Campana \& Tapia, 2017), de la cual deriva el NPT. Posterior al análisis de los inconvenientes que generan NPT se pudo encontrar el origen de dichos problemas, en este trabajo se han asociado de manera conveniente las causas a su respectivo problema. La variable causa agrupa los inconvenientes tomando en consideración el origen y el sistema mecánico al que pertenece, lo cual permite ser más específicos a la hora de clasificar y determinar la causa de un problema puesto que delimitaremos con mayor precisión la génesis de cada inconveniente. La propuesta se la puede apreciar en la Tabla 8 del Anexo 1.

\section{Diagrama causa-efecto}

Al aplicar el diagrama Ishikawa a las Tareas No Productivas, propuestas por (Ayala, Torres, Valencia, \& Loaiza, 2016), se puede identificar las causas principales y secundarias de los problemas, se ampliaron las opciones de las posibles causas de los NPT y se analizaron los procesos de perforación en búsqueda de mejoras.

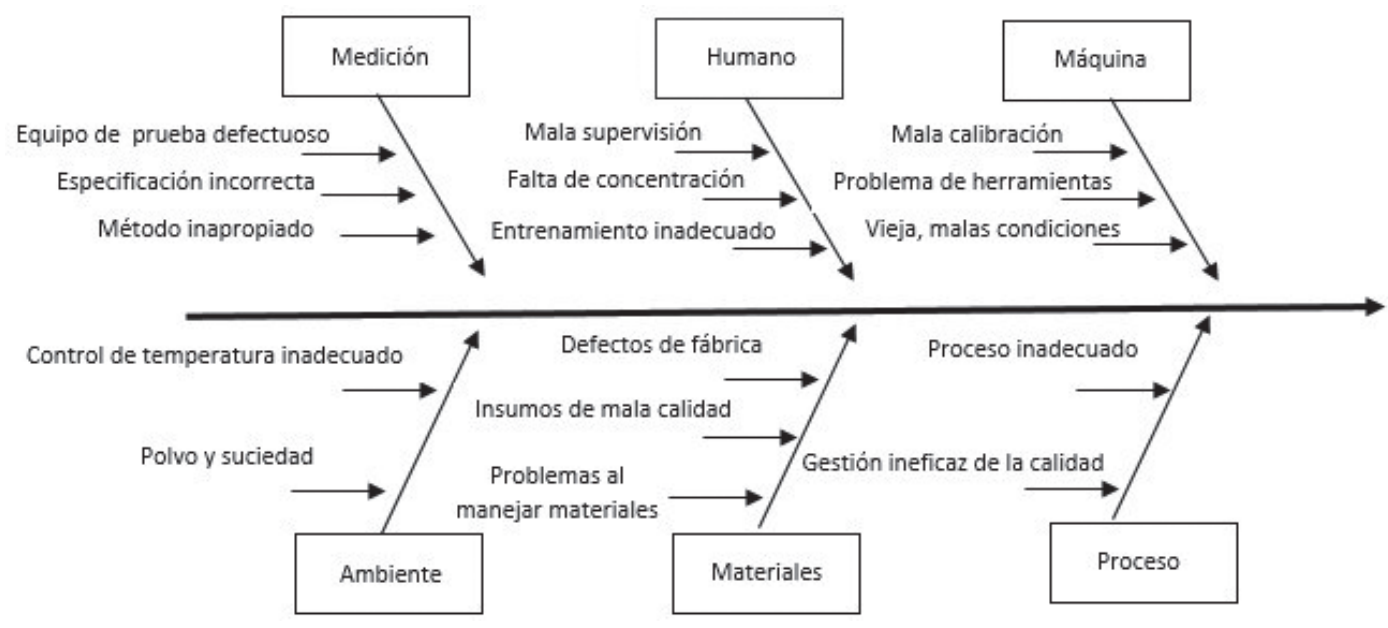

Figura 1. Diagrama causa efecto aplicado a las tareas no productivas. 


\section{Propuesta para la reducción del NPT}

Para reducir los costos existen dos alternativas, la primera enfocada en la parte técnica-operativa optimizando las operaciones, el llamado límite técnico; la segunda es la planificación de ingeniería y recae directamente sobre medidas y acciones que permitan la reducción de los NPT.

Al revisar los informes de perforación se encontró que la persona encargada de llevar el reporte clasifica los problemas bajo su propio criterio, algunas de las matrices existentes poseen una clasificación que permite catalogar un problema en más de una opción, además, se tiene el inconveniente de que cada empresa que brinda el servicio integrado de perforación posee sus propios, códigos y convenciones para registrar un NPT.

La matriz se formula bajo la consideración de que las categorías de problemas NPT sean independientes y mutuamente excluyentes, con la intensión de que guarden un orden conveniente para: mejorar la presentación, una mejor comprensión del tema por parte de los intérpretes de esta matriz y facilitar la identificación de las causas de los NPT.

\section{Descripción la matriz de NPT}

La matriz fue realizada para que los intérpretes, operadores e ingenieros, obtengan de una manera rápida y resumida la situación general del NPT, para un análisis individual o grupal de pozos perforados.

La matriz registra los valores del NPT, las frecuencias y los costos asociados a los problemas, además permite distinguir, organizar y categorizar los problemas, así como evaluar el tiempo perdido, lo cual ayudará a cuantificar el impacto económico en el proyecto de perforación.

\section{Configuración de la matriz}

Con esta configuración se pueden realizar análisis transversales que permiten observar directamente los valores de NPT parciales por tipo de problema y por secciones del pozo. Con un posterior análisis se puede determinar los costos de perforación por tipo de problema y secciones del pozo. La matriz fue formulada a partir de las tareas no productivas de las distintas empresas de servicios, tratando de que las tareas sean claras y muy bien definidas para no dar paso a errores de clasificación.
La necesidad de crear una nueva matriz NPT surge como respuesta de la problemática que implica el hecho de que cada operadora y compañía de servicio cuenten con sus categorías propias, en el estudio se observa el impacto que esto supone en los reportes finales de perforación, al encontrar categorías de problemas que provocan confusión a la hora de clasificar un problema por no encontrarse delimitadas con claridad.

La perforación es un proceso complejo, a pesar de los sistemas que integran las múltiples variables consideradas en la planificación de un pozo, aún siguen apareciendo eventos no planeados (Kaiser \& Pulshiper, 2007).

Desde el punto de vista de la gestión de proyectos, la operación de perforación debe desarrollarse cumpliendo el cronograma y el presupuesto asignado. La ocurrencia de problemas de perforación y algunas esperas técnicas y no técnicas desplazan los tiempos fuera de lo programado (Nabai, Alireza, \& Moradinejad, 2016), por todo lo expuesto esperamos que la matriz permita una organización más eficiente de los datos relevantes de los problemas al perforar.

La Tabla 1, en el Anexo 1, permite apreciar cómo se distribuyen los problemas en la matriz NPT, además de manera breve se explica el contenido que se incluye en la misma.

\section{Validación de los datos}

Es un medio que no podemos pasar por alto. La manipulación de la información desde la observación, registro y emisión del reporte, y la interpretación del problema es un proceso que puede ir restando detalles significativos, los cuales cobran relevancia a la hora de identificar las causas del NPT. La validación se llevó a cabo comparando los tiempos en sus respectivas tablas de NPT, curvas de profundidad vs. tiempo, registro de lecciones aprendidas y en los reportes diarios de perforación en las campañas de perforación comprendidas entre los años 2011 a 2014 en la Cuenca Oriente Ecuador. Para el estudio se seleccionaron únicamente los pozos de dos secciones. Los datos están disponibles en la Tabla 2, Anexo 1 al final del documento.

\section{Metodología}

Para diagnosticar el efecto dentro del proceso de perforación que tienen los NPT se realizó un análisis que deje en evidencia el incremento en el tiempo, así como en los costos finales del proyecto. 
La investigación demostró que los eventos en superficie tienen como raíz principal la falta de implementación de programas de mantenimiento preventivo y que los problemas en el fondo del pozo obedecen a fallos en los equipos como respuesta de la exposición de los mismos a condiciones agresivas de las formaciones atravesadas. Los datos recopilados se sometieron a un proceso de validación para comprobar su duración, presencia y causa; posteriormente los datos se incluyeron en la matriz de NPT, una vez registrados los tiempos se asoció a cada problema una causa, siendo este paso el aporte más relevante de la investigación. Como paso final se realizó un análisis económico del impacto del NPT en los costos de perforación.

\section{Resultados}

\section{NPT al perforar}

Los problemas aislados no reflejan el estado del NPT en los pozos, sin embargo, esta condición tampoco es motivo para que sean descartados, por el contrario, estos inconvenientes deben ser examinados para encontrar una relación con otros problemas en la sección perforada a la que corresponden, y así ver si obedecen a la misma dificultad o complejidad asociada a la geometría, profundidad y diámetro del pozo. La Figura 2 y 3 muestran los porcentajes de NPT registrados.

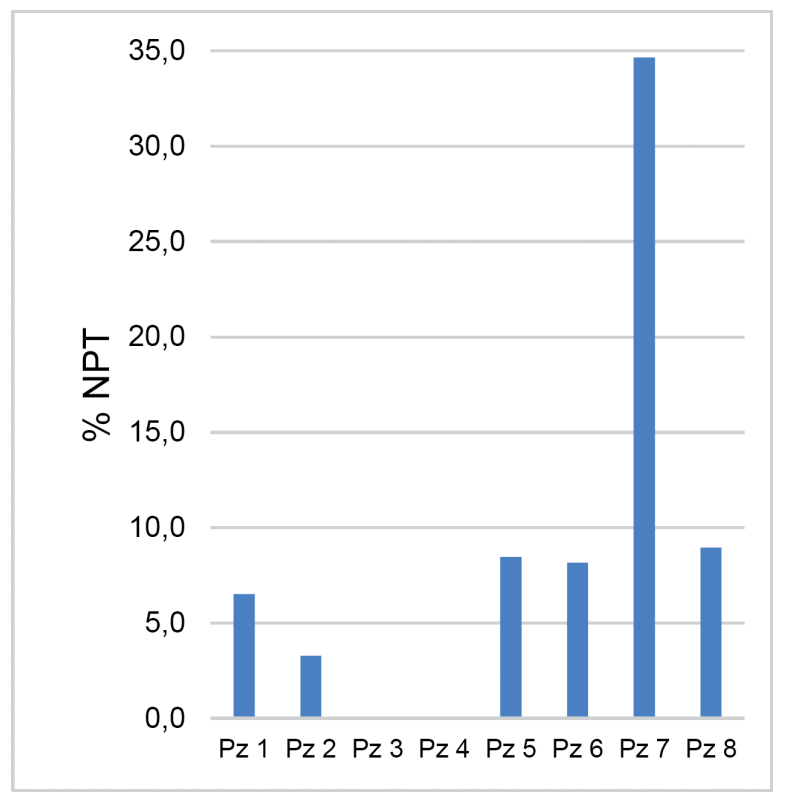

Figura 2. Porcentaje de NPT en cada pozo.

La evaluación del NPT varía entre el $6 \%$ y el $9 \%$ en 4 pozos, sobresale un caso con NPT muy alto respecto al resto que alcanza el $35 \%$. Se encuentra que en 2 pozos no se registra NPT y en uno el valor es bastante bajo del $3,27 \%$. De los 181,3 días invertidos en la perforación de los pozos 18,58 días son NPT, con un promedio de $10,26 \%$ de NPT para la muestra de 8 pozos, el detalle se observa en la Tabla 3.

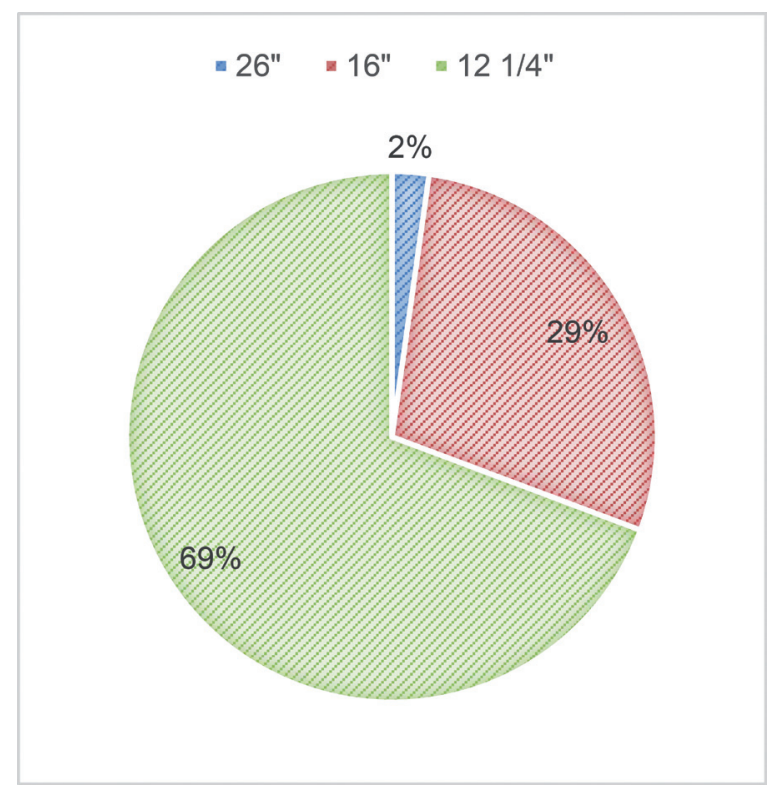

Figura 3. Porcentaje de NPT por sección.

Tabla 3. Registro del NPT en la muestra.

\begin{tabular}{|c|c|c|c|c|c|c|c|}
\hline $\begin{array}{c}\text { Pozo } \\
\mathbf{N}^{\circ}\end{array}$ & Tipo de pozo & $\begin{array}{l}\text { Sección } \\
\text { final }\end{array}$ & MD & $\begin{array}{l}\text { Perforación } \\
\text { Días }\end{array}$ & $\begin{array}{l}\text { NPT } \\
\text { Horas }\end{array}$ & $\begin{array}{l}\text { NPT } \\
\text { Días }\end{array}$ & $\begin{array}{c}\text { NPT } \\
\%\end{array}$ \\
\hline Pz 1 & Direccional & $121 / 4^{\prime \prime}$ & 10360 & 18,9 & 29,5 & 1,2 & 6,5 \\
\hline Pz 2 & $\begin{array}{c}\text { Direccional } \\
\text { Tipo S }\end{array}$ & $121 / 4^{\prime \prime}$ & 9965 & 19,7 & 15,5 & 0,6 & 3,3 \\
\hline Pz 3 & $\begin{array}{c}\text { Direccional } \\
\text { Tipo S }\end{array}$ & $121 / 4 "$ & 10651 & 22,4 & 0,0 & 0,0 & 0,0 \\
\hline $\mathrm{Pz} 4$ & $\begin{array}{c}\text { Direccional } \\
\text { Tipo S }\end{array}$ & $121 / 4 "$ & 10575 & 20,0 & 0,0 & 0,0 & 0,0 \\
\hline Pz 5 & $\begin{array}{l}\text { Direccional } \\
\text { Tipo S }\end{array}$ & $121 / 4^{\prime \prime}$ & 9996 & 20,2 & 41,0 & 1,7 & 8,5 \\
\hline Pz 6 & $\begin{array}{c}\text { Direccional } \\
\text { Tipo S }\end{array}$ & $121 / 4 "$ & 10183 & 23,5 & 46,0 & 1,9 & 8,2 \\
\hline Pz 7 & $\begin{array}{c}\text { Direccional } \\
\text { Tipo S }\end{array}$ & $121 / 4 "$ & 10174 & 31,3 & 260,0 & 10,8 & 34,6 \\
\hline \multirow[t]{2}{*}{ Pz 8} & $\begin{array}{c}\text { Direccional } \\
\text { Tipo S }\end{array}$ & $121 / 4 "$ & 10711 & 25,2 & 54,0 & 2,3 & 8,9 \\
\hline & & & Total & 181,1 & 446,0 & 18,6 & 10,3 \\
\hline
\end{tabular}

La mayoría de los pozos se perforó en 2 secciones y el resto en 3 secciones siendo estos últimos en donde se aprecia que aumenta el NPT. 
Todos los pozos han sido perforados con taladros de capacidad 2000 HP hasta la sección final de 12 1/4", es primordial destacar que no se observa evidencia de relación entre la profundidad perforada y el NPT, descartando así una condición que es típica en otros estudios. Como muestra de esa excepción se mencionará que el pozo con mayor profundidad en la campaña 2015 se perfora sin registrar NPT.

La distribución del NPT por secciones muestra que el mayor porcentaje se concentra en la sección final de 12 1/4" con el 69,28\% mientras en la sección de 16 " se encuentra el 28,48 \% del NPT. La sección de 26 " que se encuentra en 3 pozos tan solo suma el $2,24 \%$ del NPT, esto indica que en los pozos con 3 secciones de perforación no existe un aumento del NPT de forma importante debido a esta sección adicional.

Para detectar las dificultades de la operación de perforación a partir del NPT es importante considerar la frecuencia y distribución de los eventos de fallas en el grupo de pozos. De acuerdo con esto se puede estimar los problemas más importantes en cada sección según se ha organizado la información. Se considerarán dos términos para detallar los eventos NPT: frecuencia, se considerará al número de veces que un evento ocurre en un mismo pozo. Distribución, hará referencia al número de veces que un evento se repite en los pozos de la muestra.

El número de eventos relacionados con NPT en la sección de 16" tiene una frecuencia de 18 eventos y de 12 en la sección de 12 1/4”.

\section{Sección de 16"}

El problema más frecuente dentro del pozo es falla del BHA - herramientas, impidiendo efectuar un trabajo normal por la avería o falta de respuesta de un equipo que lo compone, el problema está distribuido en 4 de los 6 pozos que presentaron NPT. Considerando el criterio de distribución y frecuencia, el segundo problema en importancia es reparaciones en el taladro, que se presenta en 4 eventos y en 3 pozos. Otros problemas destacables son problemas TDS (Top Drive System), falla de revestimientos, esto incluye dificultades en el proceso de corrida de las tuberías, fallos de los equiposaccesorios, fallas de los revestimientos después de incorporados en el pozo, y limpieza de línea de retorno que se presentan en 2 pozos. La Tabla 4, presenta el detalle del NPT.
Tabla 4. NPT en la sección de 16".

\begin{tabular}{|cccc}
\hline Problema & $\begin{array}{c}\text { Tiempo } \\
\text { NPT horas }\end{array}$ & Frecuencia & Distribución \\
\hline $\begin{array}{c}\text { Falla del BHA }- \\
\text { herramientas }\end{array}$ & 32 & 4 & 4 \\
\hline $\begin{array}{c}\text { Reparaciones en el } \\
\text { taladro }\end{array}$ & 32 & 4 & 3 \\
\hline $\begin{array}{c}\text { Problemas TDS } \\
\text { Falla de revestimientos }\end{array}$ & 15,5 & 3 & 2 \\
\hline $\begin{array}{c}\text { Limpieza de la línea de } \\
\text { retorno }\end{array}$ & 5 & 2 & 2 \\
\hline $\begin{array}{c}\text { Pega de Tubería } \\
\text { Limpieza y rimado del } \\
\text { hoyo }\end{array}$ & 29 & 2 & 2 \\
\hline
\end{tabular}

\section{Sección 12 1/4"}

Aquí se presentan problemas de reparaciones en el taladro, se encuentran distribuidos en diferentes pozos. Una gran cantidad de NPT corresponde a inconvenientes en el pozo, específicamente el problema de limpieza y rimado del hoyo. El otro contratiempo destacable dentro del pozo es falla del BHA - herramientas. El detalle del NPT se tabula en la Tabla 5.

Tabla 5. NPT en la sección de 12 1/4”.

\begin{tabular}{cccc}
\hline Problema & $\begin{array}{c}\text { Tiempo } \\
\text { NPT horas }\end{array}$ & Frecuencia & Distribución \\
\hline $\begin{array}{c}\text { Limpieza y rimado del } \\
\text { hoyo }\end{array}$ & 184 & 4 & 2 \\
\hline $\begin{array}{c}\text { Reparaciones en el } \\
\text { taladro }\end{array}$ & 17,5 & 3 & 3 \\
\hline $\begin{array}{c}\text { Falla del BHA - } \\
\text { herramientas }\end{array}$ & 48,5 & 2 & 2 \\
\hline Falla de revestimientos & 54,5 & 1 & 1 \\
\hline
\end{tabular}

\section{Estado del NPT en superficie y en el pozo}

\section{En superficie}

El análisis se enfoca en el NPT que ocurre en la torre de perforación (taladro), el mismo que se distribuye principalmente entre los siguientes problemas: reparaciones en el taladro, problemas TDS y limpieza de la línea de retorno. La variable reparaciones en el taladro está presente en las 2 secciones consideradas y corresponde a un $25 \%$ de un total de $28,48 \%$ de NPT registrado en la sección de 16 ", lo que convierte a este problema en un inconveniente reiterado. La frecuencia de NPT en superficie (torre de perforación) en la sección de 16 " es la que predomina y está distribuida en 5 de 
los 8 pozos de la muestra. En la sección 12 1/4" existen 3 eventos de reparaciones en el taladro distribuidos en 3 pozos de los 8 en estudio.

Se ratifica que los problemas principales en superficie se enfocan en la torre de perforación, y el evento preponderante es reparaciones en el taladro, que se presenta en 4 pozos en las 2 secciones de perforación con 7 eventos y un total de 49,5 horas. Otra dificultad son los problemas TDS que se presenta en 3 pozos, solamente en la sección de 16", y con 15,5 horas de NPT. En los pozos 1,2 y 5 se observa mayor cantidad de NPT en superficie. La Figura 4 representa el NPT en superficie.

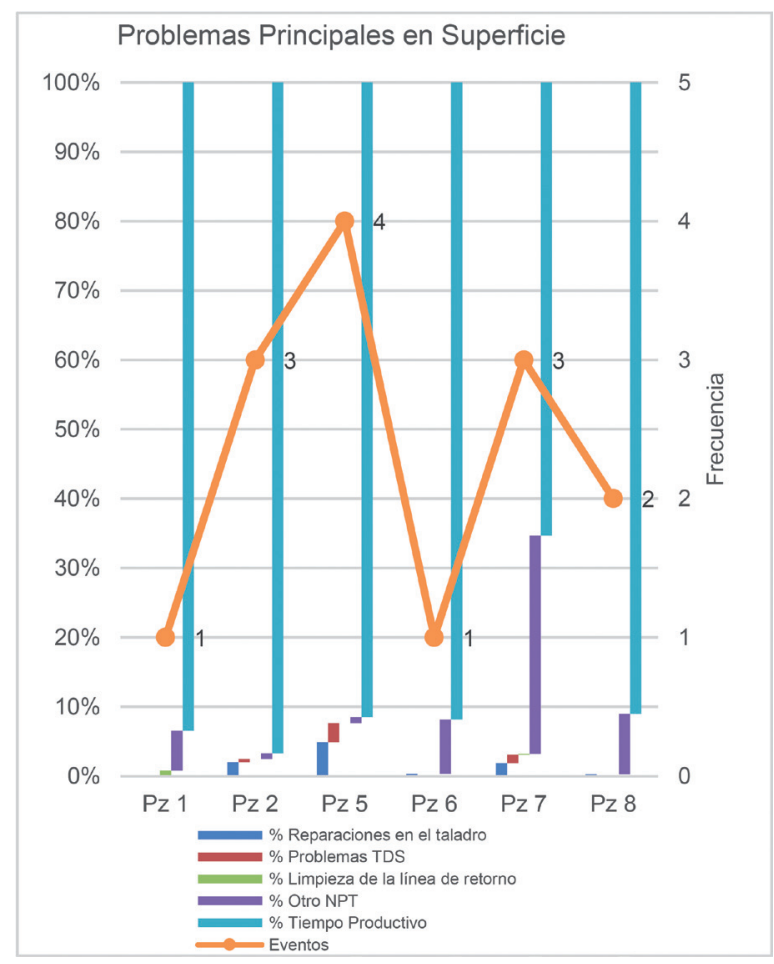

Figura 4. NPT en superficie.

\section{En el pozo}

$\mathrm{Al}$ enfocarse en los problemas en el interior del pozo la falla del BHA - herramientas se presenta en un $25 \%$ del $28,48 \%$ del NPT registrado en la sección de 16 " y un $15 \%$ del $69,28 \%$ del NPT de la sección 12 1/4". Se debe acotar que el NPT se concentra en la sección de $12 \frac{1}{4}$ ", lo cual obedece a un comportamiento típico de la perforación al avanzar en profundidad y complejidad geométrica.

La limpieza y rimado del hoyo se distribuye en la sección de 12 1/4" y de manera proporcional y en menor volumen se distribuye la falla de revestimientos en las secciones 16 " y 12 1/4". La gran cantidad de NPT en la sección de 12 1/4" se distribuye solamente en 3 pozos.

El problema falla del BHA - herramientas es el más significativo, puesto que se halla distribuido en 5 pozos con 6 eventos, ocupando la importante cantidad de tiempo de 80,5 horas, distribuidas entre los diferentes pozos y representa el $18 \%$ del NPT.

La limpieza y rimado del hoyo tiene una frecuencia de 5 eventos con 191,5 horas que representa el $43 \%$ de NPT, se presenta en 3 pozos con eventos persistentes. El evento falla de revestimientos se registraron 3 eventos en 3 pozos, el NPT se concentra en uno de ellos con 59,5 horas. La Figura 5, muestra el NPT en el pozo.

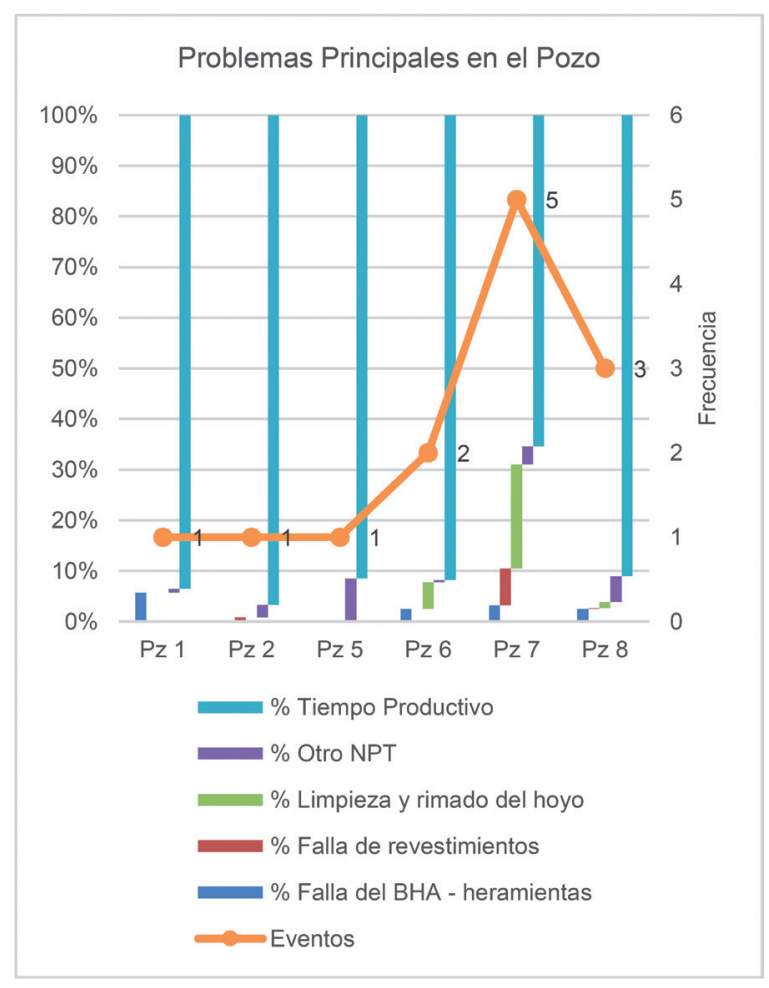

Figura 5. NPT en el fondo del pozo.

\section{Discusión}

\section{Causas de los problemas principales}

Posterior a la revisión de los eventos de fallas en el pozo, se observa que estos problemas obedecen a la complejidad que presenta la formación (geología regional) para las operaciones, muestra de ello es el deterioro de los elementos del BHA producido por vibración y abrasión que presenta la formación Napo, así como la complejidad de avanzar con la perforación en condiciones de hueco apretado y empaquetamientos que presenta la formación Tena. 
No se verifica que los problemas hayan sido influenciados por prácticas inadecuadas en los procedimientos ejecutados por el personal. Las causas de los problemas se resumen en la Tabla 6 .

Tabla 6. Causas de los problemas principales que generan NPT.

\begin{tabular}{cccc}
\hline Causas & Problemas & Horas & $\%$ \\
\hline Fallas en el Taladro & $\begin{array}{c}\text { Reparaciones en el taladro; } \\
\text { problemas TDS }\end{array}$ & 79,5 & 17,83 \\
\hline $\begin{array}{c}\text { Complejidad Pozo- } \\
\text { Formación }\end{array}$ & $\begin{array}{c}\text { Falla del BHA - herramientas; } \\
\text { limpieza y rimado del hoyo; } \\
\text { falla de revestimientos }\end{array}$ & 332 & 74,44 \\
\hline Organización & Esperas & 1,5 & 0,34 \\
\hline-- & Problemas dispersos & 33 & 7,40 \\
\hline
\end{tabular}

\section{Costo asociado al NPT}

El análisis se realiza después de categorizar los servicios con costos fijos y los servicios con costos variables, siendo éstos últimos los que dependen del tiempo y aquellos que influyen en el costo final del proyecto. Por esa razón se calculará el valor adicional de cada pozo como consecuencia del NPT, la Tabla 7, describe la clasificación.

Tabla 7. Costos fijos y variables en perforación.

\begin{tabular}{cc}
\hline VARIABLES & FIJOS \\
\hline Fluidos de perforación (FP) & Brocas \\
\hline Perforación direccional (PD) & Cementación \\
\hline Control litológico (CL) & Otros cargos \\
\hline Rol de compañía (RC) & Registros eléctricos \\
\hline Supervisor de perforación (Co man) & Tubería de revestimiento \\
\hline Asistente de perforación & Cabezal de producción \\
\hline
\end{tabular}

Haciendo uso de la ecuación (1) propuesta por (Bonilla \& Buestán, 2013) se determina los costos variables (costo / día) en los servicios que dependen del tiempo y estos son:

- Fluidos de perforación (FP)

- Perforación direccional (PD)

- Control litológico (CL)

- Rol de compañía (RC)

$$
\mathrm{CPD}=\frac{\mathrm{FP}+\mathrm{PD}+\mathrm{CL}+\mathrm{RC}}{\text { Tiempo de operaciones }}
$$

Reducir el NPT implica incrementar la productividad, un activo con mayor productividad costará menos operar. (Athenes Group, 2015).

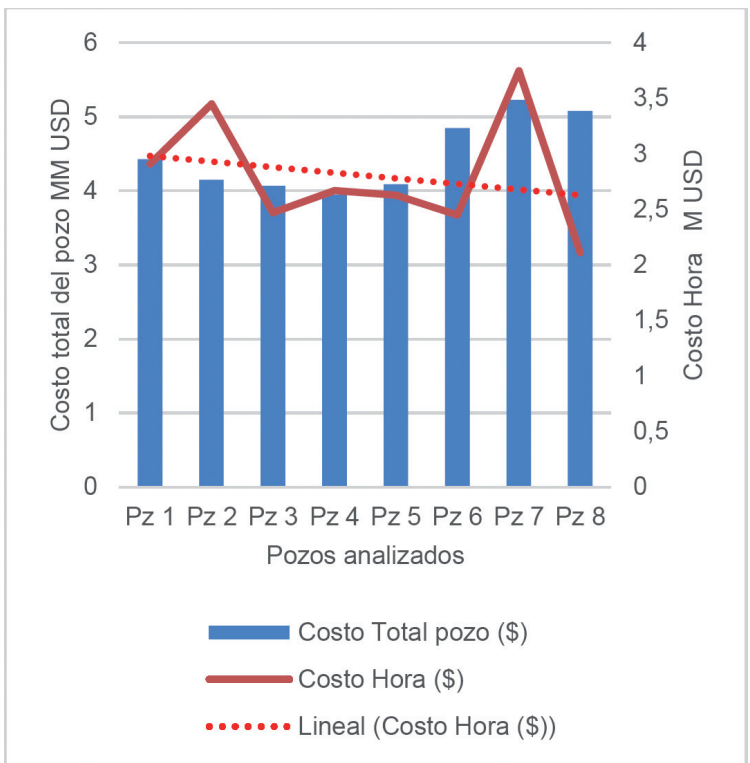

Figura 6. Costo variable total y por hora.

La Figura 6, expone los resultados obtenidos. Los pozos 2, 3, 4 y 7, muestran los costos variables más altos. Por otra parte, con una tendencia uniforme los costos más bajos se observan en los pozos $1,4,6,8$. Los resultados guardan concordancia con lo obtenido en el análisis técnico, demostrando el efecto directo en el costo que tienen las operaciones no productivas. El detalle del costo variable por secciones, así como el registro del costo por problema se lo puede apreciar en la matriz Tabla 2, la cual fue completada con los valores determinados en esta parte del estudio. En términos generales podemos decir que la campaña de perforación de los 8 pozos fue exitosa. Para llegar a esta conclusión, se ha tomado el costo promedio de 3000 USD/HORA propuesto por Schlumberger, un valor estándar general (utilizado para cualquier taladro, y para cualquier geometría de pozo), cifra referencial para determinar el costo del tiempo asociado a las tareas no productivas que generan NPT.

Schlumberger, no indica en ninguna fuente bibliográfica cómo se obtiene este costo (genérico único), tampoco explica por qué lo usa de manera indistinta para cualquier geometría de pozo. Al parecer es solo un valor referencial que ayuda a tener una idea del costo/ hora. Por esta razón, al comparar los valores obtenidos en los cálculos, salvo dos excepciones (pozo 2 y 7 ) que su costo/hora excede los 3000 USD/HORA, se puede decir que la perforación mantiene niveles aceptables de NPT. La suma acumulada del costo asociada a las tareas no productivas fue de 908 212,5 USD, un valor nada despreciable en la situación económica actual de la industria. 


\section{NPT como medida para evaluar la optimización de la perforación}

La tasa de perforación (días/pie) suele ser una de las variables más utilizadas para verificar la optimización del proceso de perforación, ilustrada en la Figura 7. El NPT es una variable dependiente del tiempo, enfocado en las tareas no productivas y en los costos variables, puede ser un excelente apoyo de la planeación y una herramienta de diagnóstico para determinar el éxito de un proyecto.

En la muestra se contrastó el valor del NPT vs. la tasa de perforación. En la Figura 7 se observa que la tasa de perforación mantiene una constante aproximada de 0,2 días/pie perforado, sin embargo la curva del NPT nos hace notar que el pozo 7, a pesar de encontrarse dentro de la media por cada 100 pies perforados su NPT es el más alto, lo que permite concluir que esta variable no es del todo infalible, y por esta razón, el análisis del NPT podría revelar eventos no considerados, relegados por lo diversos y dispersos que se encuentran en el proceso, pero que al sumarlos significan un impacto considerable en el tiempo final.

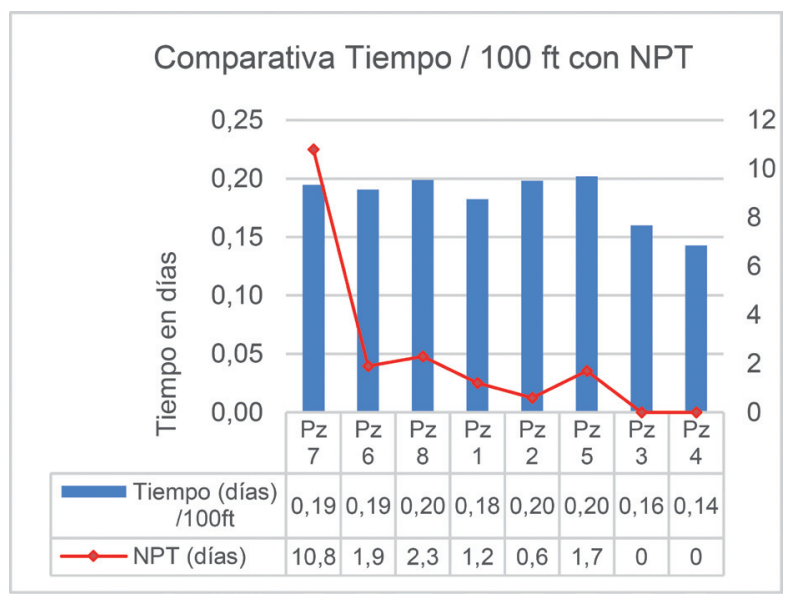

Figura 7. Análisis de tasa de perforación y el NPT.

\section{Conclusiones y recomendaciones}

La muestra de pozos con profundidad aproximada de 10000 pies MD, evidencia que en los pozos de dos secciones presentan una acumulación de NPT con respecto a los pozos de tres de secciones, lo que permite establecer que existe una relación directa entre el NPT y el número de secciones que se planifiquen para un pozo.

El NPT puede revelar que las tasas de perforación no siempre permiten diagnosticar con certeza absoluta que un pozo se ha desarrollado con éxito, el NPT permite revelar problemas aislados o recurrentes que por su duración podrían ser descartados o desestimados.

Existe presencia de eventos aislados, pero de larga duración como son pega de tubería, limpieza y rimado del hoyo, los cuales deben ser analizados de manera separada para no afectar la evaluación final de toda la campaña de perforación realizada.

La aplicación de la matriz de NPT permitió consolidar la duración, la frecuencia de aparición de un evento, así como el costo asociado a cada tarea no productiva por secciones y el costo total del NPT de una campaña de perforación, lo cual agilitó el análisis y el diagnóstico de los problemas más importantes.

Establecer las causas más comunes de los problemas que generan NPT permitió establecer el origen de las tareas no productivas, con este recurso se pudo establecer un requerimiento puntual de mantenimiento preventivo.

Los problemas en superficie son los que más NPT generan específicamente problemas TDS, reparaciones en el taladro y limpieza en la línea de retorno, todos ellos tienen una génesis común, los fallos registrados en el taladro, estos problemas se hacen más recurrentes en la sección de 16» y están asociados a la falta de mantenimiento preventivo.

La muestra de ocho pozos reúne un total de 446 horas de NPT a la cual se asocia un costo de 908212 USD, el valor calculado del costo/hora NPT fue de 2000 USD, valor que se encuentra por debajo del costo promedio que establece Schlumberger para cada hora de NPT, el cual es de 3000 USD/hora, lo que nos permite definir a la campaña de perforación como un proceso exitoso.

\section{Referencias}

1. Adams, N. (1977). A Field Case Study of DifferentialPressure Pipe. SPE Annual Fall Technical Conference and Exhibition, SPE-6716-MS, Denver Colorado, USA (8).

2. Alappat, N. (2015). Linkened. Obtenido de htpp:// www.linkened.com/pulse/measuring-nonproductive-time-kpi-minimize-drilling-flatalappat/

3. Athenes Group. (2015). Beyond Non Productive Time (NPT): Effective Production Time. Obtenido de http://www.athensgroup.com/ news/beyond-non-productive-time-npteffective-production-time/. 
4. Ayala, D., Benítez, A., \& Valencia, R. (2017). Optimización de la Tasa de Penetración mediante el análisis de las vibraciones al perforar, caso de estudio Ecuador. Revista Fuentes, 15(1), 27-40.

5. Ayala, F. E. B., Gómez, J. Q., \& León, E. A. (2011). Estudio de factibilidad del uso del biodiesel como fase contínua en lodos de perforación de emulsión inversa. Fuentes: El reventón energético, 9(1), 6 .

6. Ayala, D., Torres, F., Valencia, R., \& Loaiza, M. (2016). Impacto del Tiempo no Productivo (NPT) en Operaciones de Perforación y Análisis de los Datos Mediante la Prueba de Chicuadrado. Fuentes El Reventón Energético (11).

7. Bonilla, M., \& Buestán, A. (2013). Estudio del Límite Técnico para la Perforación de Pozos en los Campos Cuyabeno y VHR. Quito: EPN (316).

8. Campana, D. E. A., \& Tapia, R. A. V. (2017). Evaluación cualitativa de la limpieza de hoyo en pozos de alta inclinación-alto desplazamiento en la Cuenca Oriente. Fuentes: El reventón energético, 15(2), 49-56.

9. Halliburton. (2013). Halliburton. Obtenido de http:// www.halliburton.com/en-US/ps/solutions/ deepwater/challenges-solutions/reduce-nonroductive-time.page?node-id=hgjyd452\&Topi $\mathrm{c}=$ DeepwaterWestAfrica.

10. Kaiser, M., \& Pulshiper, A. (2007). Generalized Functional Models for Drilling Cost Estimation. SPE Drilling \& Completion, SPE98401-PA. (7).

11. Moazzeni, A., Nabaei, M., \& Azari, A. (2011). Reducing Consumed Energy while Drilling an Oil Well through a Deep. Omidihe Iran:
Advanced in Petroleum Exploration and Development, Omidiech, Iran (10).

12. Molina, D. (2014). Modelo de Control de Costos de Perforación de Pozos para los Campos Cuyabeno-VHR de Petroamazonas EP. Quito: Universidad Tecnológica Israel (152).

13. Morán, H. (2014). Análisis del Proceso de Perforación Direccional de Pozos Petroleros en el campo Auca de la Amazonía Ecuatoriana para la Reducción de los Tiempos no Productivos. Quito: UTE (217).

14. Nabai, M., Alireza, M., \& Moradinejad, M. (2016). Non-Productive Time (NPT) Analysis, New Opportunity in Drilling Operation Management. Rama Omidieh: Azad Islamic University (7).

15. Portilla, H. E., Suárez, D. F., \& Corzo, R. (2012). Metodología para la optimización de parámetros de perforación a partir de propiedades geomecánicas. Revista Fuentes, 10(2).

16. Weakley, R. (1990). Use of Stuck Pipe Statistics to Reduce the Occurrence of Stuck Pipe. SPE Annual Technical Conference and Exhibition, SPE-20410, New Orleans, Louisiana (8).

17. Wisnie, A., \& Zhiwei, Z. (1994). Quantifying Stuck Pipe Risk in Gulf of Mexico Oil and Gas Drilling. SPE Annual Technical Conference and Exhibition, SPE-28298-MS, New Orleans, Louisiana (12).

18. Zapata, I. (2011). Evaluación de Tiempos productivos e Improductivos en las Operaciones de los Taladros o Cabrias de RA/RC de la Empresa San Antonio Internacional durante los Años 2009-2010. Puerto la Cruz: Universidad de Oriente (118). 


\section{ANEXO 1}

Tabla 1. Descripción de la matriz NPT.

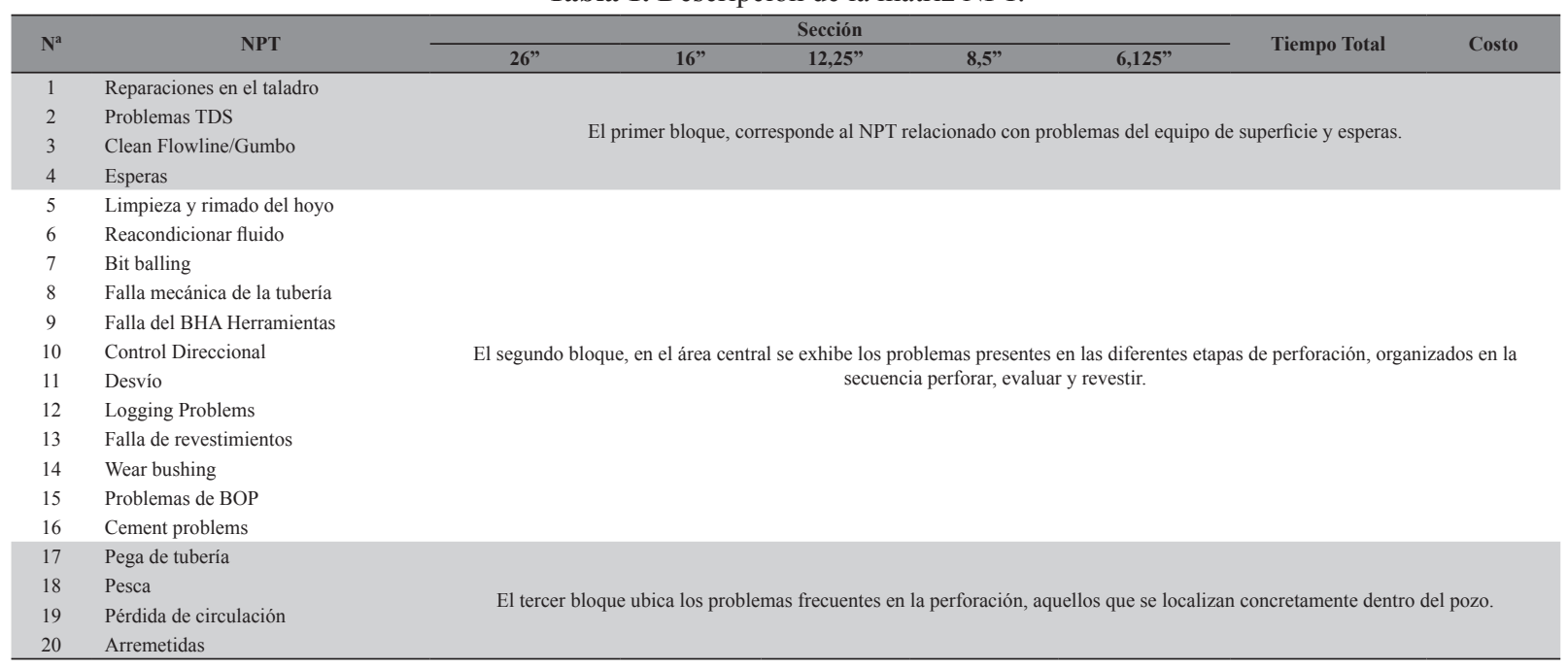

Tabla 2. Matriz NPT de la muestra en estudio.

\begin{tabular}{|c|c|c|c|c|c|c|c|c|}
\hline \multirow{3}{*}{ NPT } & \multicolumn{6}{|c|}{ Secciones } & \multirow{3}{*}{ Total, horas } & \multirow{3}{*}{ Costo USD } \\
\hline & \multicolumn{2}{|c|}{$26 "$} & \multicolumn{2}{|c|}{$16 "$} & \multicolumn{2}{|c|}{$12 \frac{1 / 4 "}{}$} & & \\
\hline & Hrs & \# Eventos & Hrs & \# Eventos & Hrs & \# Eventos & & \\
\hline \multirow{2}{*}{ Reparaciones en el taladro } & 1 & 1 & 32 & 4 & 18 & 3 & 51 & 1032750 \\
\hline & & & \multicolumn{2}{|c|}{$\$ 64800$} & \multicolumn{2}{|c|}{$\$ 36450$} & 51 & $103.275,0$ \\
\hline \multirow{2}{*}{ Problemas TDS } & 9 & 1 & 16 & 3 & 0 & 0 & & \\
\hline & & & \multicolumn{2}{|c|}{$\$ 32400$} & \multicolumn{2}{|c|}{-- } & 25 & $50.625,0$ \\
\hline \multirow{2}{*}{ Limpieza de la línea de retorno } & 0 & 0 & 4,5 & 2 & 0 & 0 & 4.5 & 91125 \\
\hline & & & \multicolumn{2}{|c|}{$\$ 9112,5$} & & & 4,5 & $9.112,5$ \\
\hline \multirow{2}{*}{ Esperas } & 0 & 0 & 0 & 0 & 1,5 & 1 & & \\
\hline & & & \multicolumn{2}{|c|}{-- } & \multicolumn{2}{|c|}{$\$ 3037,5$} & 1,5 & $3.037,5$ \\
\hline \multirow{2}{*}{ Limpieza y rimado del hoyo } & 0 & 0 & 7,5 & 1 & 184 & 4 & & \\
\hline & & & \multicolumn{2}{|c|}{$\$ 15187,5$} & \multicolumn{2}{|c|}{$\$ 372600$} & 192 & $388.800,0$ \\
\hline \multirow{2}{*}{ Reacondicionar lodo } & 0 & 0 & 0 & 0 & 0 & 0 & 0 & 00 \\
\hline & & & & & & & 0 & 0,0 \\
\hline & 0 & 0 & 0 & 0 & 0 & 0 & & \\
\hline Embolamiento de la broca & & & & & & & 0 & 0,0 \\
\hline & 0 & 0 & 0 & 0 & 0 & 0 & & \\
\hline Falla mecánica de la tubería & & & & & & & 0 & 0,0 \\
\hline & 0 & 0 & 32 & 4 & 49 & 2 & & \\
\hline Falla del BHA herramientas & & & & & & & 81 & $164.025,0$ \\
\hline & 0 & 0 & 0 & 0 & 0 & 0 & & \\
\hline Control direccional & & & & & & & 0 & 0,0 \\
\hline & 0 & 0 & 0 & 0 & 0 & 0 & & \\
\hline Desvío & & & & & & & 0 & 0,0 \\
\hline Problemas de reoistros & 0 & 0 & 0 & 0 & 3 & 1 & 3 & 60750 \\
\hline Problemas de registros & & & & & & & 3 & $6.075,0$ \\
\hline Follas de reyectimiento & 0 & 0 & 5 & 2 & 55 & 1 & & \\
\hline Fallas de revestimiento & & & & & & & 60 & $121.500,0$ \\
\hline Problemas del cahezal & 0 & 0 & 0 & 0 & 0 & 0 & 0 & \\
\hline & & & & & & & 0 & 0,0 \\
\hline & 0 & 1 & 1,5 & 0 & 0 & 0 & & \\
\hline Problemas de BOP & & & & & & & 1,5 & $3.037,5$ \\
\hline Problemas de cementación & 0 & 0 & 0 & 0 & 0 & 0 & 0 & 0.0 \\
\hline Problemas de cementacion & & & & & & & 0 & 0,0 \\
\hline & 0 & 0 & 29 & 1 & 0 & 0 & & \\
\hline Pega de tubería & & & & & & & 29 & $58.725,0$ \\
\hline Pesca & 0 & 0 & 0 & 0 & 0 & 0 & 0 & 00 \\
\hline Pesca & & & & & & & 0 & 0,0 \\
\hline Pérdida de circulación & 0 & 0 & 0 & 0 & 0 & 0 & 0 & 00 \\
\hline Pérdida de circulación & & & & & & & 0 & 0,0 \\
\hline Arremetidas & 0 & 0 & 0 & 0 & 0 & 0 & 0 & 0.0 \\
\hline Arremetidas & & & & & & & 0 & 0,0 \\
\hline & 10 & & 127 & & 309 & & & \\
\hline Total & & & & & & & 446 & $908.212,5$ \\
\hline
\end{tabular}


Tiempo no productivo en pozos de dos secciones, caso de estudio Ecuador

Tabla 3. Clasificación de las tareas no productivas y las causas más comunes que las provocan.

\begin{tabular}{|c|c|c|c|c|c|c|c|c|c|c|}
\hline Problema & Causa 1 & Causa 2 & Causa 3 & Causa 4 & Causa 5 & Causa 6 & Causa 7 & Causa 8 & Causa 9 & Causa 10 \\
\hline $\begin{array}{l}\text { Reparaciones en el } \\
\text { taladro }\end{array}$ & Bombas de lodo & Generador & Malacate & $\begin{array}{c}\text { Stand pipe / } \\
\text { manguerote/cuello } \\
\text { de ganso / líneas de } \\
\text { descarga }\end{array}$ & Manifold & Gato hidráulico & $\begin{array}{l}\text { Llaves } \\
\text { lagartos }\end{array}$ & Llaves st 80 & & \\
\hline Problemas TDS & $\begin{array}{l}\text { Bomba de } \\
\text { transmisión }\end{array}$ & $\begin{array}{l}\text { Tarjeta de } \\
\text { control }\end{array}$ & $\begin{array}{l}\text { Cambio de } \\
\text { mordazas del } \\
\text { pipe hanger }\end{array}$ & Sistema electrónico & $\begin{array}{l}\text { Cambio de } \\
\text { wash pipe }\end{array}$ & $\begin{array}{c}\text { Sistema } \\
\text { mecánico } \\
\text { engranes/caja } \\
\text { de cambio }\end{array}$ & $\begin{array}{c}\text { Sistema } \\
\text { hidráulico }\end{array}$ & $\begin{array}{l}\text { Sistema } \\
\text { eléctrico }\end{array}$ & Brazo elevador & $\begin{array}{l}\text { Unidad de } \\
\text { potencia }\end{array}$ \\
\hline $\begin{array}{l}\text { Clean Flowline/ } \\
\text { Gumbo }\end{array}$ & $\begin{array}{c}\text { Arcilla blanda } \\
\text { pegajosa Gumbo }\end{array}$ & $\begin{array}{c}\text { Diámetro flow } \\
\text { line inadecuado }\end{array}$ & & & & & & & & \\
\hline Esperas & Falla de logística & $\begin{array}{l}\text { No está en stock } \\
\text { el repuesto }\end{array}$ & $\begin{array}{c}\text { No } \\
\text { disponibilidad } \\
\text { inmediata del } \\
\text { técnico }\end{array}$ & & & & & & & \\
\hline $\begin{array}{l}\text { Limpieza y rimado } \\
\text { del hoyo }\end{array}$ & $\begin{array}{l}\text { Arcillas } \\
\text { reactivas }\end{array}$ & $\begin{array}{l}\text { Mala limpieza } \\
\text { de casing }\end{array}$ & No baja casing & & & & & & & \\
\hline $\begin{array}{l}\text { Reacondicionar } \\
\text { fluido }\end{array}$ & $\begin{array}{c}\text { Punto de } \\
\text { asentamiento no } \\
\text { planeado }\end{array}$ & $\begin{array}{l}\text { Lodo cortado } \\
\text { por gas }\end{array}$ & $\begin{array}{c}\text { Fluido } \\
\text { contaminado } \\
\text { por acuífero }\end{array}$ & $\begin{array}{l}\text { Disminución de } \\
\text { densidad del lodo }\end{array}$ & & & & & & \\
\hline Bit balling & $\begin{array}{l}\text { Arcillas } \\
\text { reactivas }\end{array}$ & & & & & & & & & \\
\hline $\begin{array}{l}\text { Falla mecánica de } \\
\text { la tubería }\end{array}$ & Falla en rosca & Desconexión & Colapso & Estallido & Washout & Pandeo & $\begin{array}{c}\text { Tensión / } \\
\text { torsión }\end{array}$ & & & \\
\hline $\begin{array}{l}\text { Falla del BHA } \\
\text { Herramientas }\end{array}$ & $\begin{array}{l}\text { Recepción de } \\
\text { señal }\end{array}$ & $\begin{array}{c}\text { Broca } \\
\text { (mecánica/ } \\
\text { conos / jets }\end{array}$ & $\begin{array}{l}\text { MWD } \\
\text { Calibración/ } \\
\text { válvula/ } \\
\text { programa }\end{array}$ & $\begin{array}{l}\text { LWD Calibración/ } \\
\text { válvula/programa }\end{array}$ & Motor de fondo & $\begin{array}{c}\text { Fuga en sellos/ } \\
\text { empaques } \\
\text { motores de } \\
\text { fondo }\end{array}$ & & & & \\
\hline $\begin{array}{l}\text { Control } \\
\text { Direccional }\end{array}$ & BHA no desliza & $\begin{array}{c}\text { Poca respuesta } \\
\text { direccional }\end{array}$ & $\begin{array}{l}\text { Inadecuada } \\
\text { configuración } \\
\text { de BHA }\end{array}$ & $\begin{array}{l}\text { Suministro de energía } \\
\text { cable/conectividad }\end{array}$ & & & & & & \\
\hline Desvío & Pesca fallida & $\begin{array}{c}\text { Vibraciones en } \\
\text { la sarta }\end{array}$ & $\begin{array}{l}\text { Complejidad } \\
\text { geológica }\end{array}$ & & & & & & & \\
\hline Logging Problems & Baterías & Cable & & & & & & & & \\
\hline $\begin{array}{l}\text { Falla de } \\
\text { revestimientos }\end{array}$ & $\begin{array}{l}\text { Empacadura } \\
\text { defectuosa }\end{array}$ & $\begin{array}{l}\text { Lutita } \\
\text { solidificada en } \\
\text { caja, cuñas de } \\
\text { colgador }\end{array}$ & $\begin{array}{l}\text { Casing drive } \\
\text { system }\end{array}$ & $\begin{array}{l}\text { Acondicionar tope de } \\
\text { colgador }\end{array}$ & $\begin{array}{l}\text { Tubería } \\
\text { colapsada } \\
\text { ligeramente }\end{array}$ & $\begin{array}{l}\text { Tubería rota en } \\
\text { área de cabezal }\end{array}$ & & & & \\
\hline Wear bushing & $\begin{array}{l}\text { Falla al instalar } \\
\text { (torque) }\end{array}$ & & & & & & & & & \\
\hline Problemas de BOP & $\begin{array}{l}\text { Sello de boca de } \\
\text { pozo con liqueo }\end{array}$ & $\begin{array}{l}\text { Liqueo en líneas } \\
\text { hidráulicas }\end{array}$ & $\begin{array}{l}\text { Válvula } \\
\text { reguladora } \\
\text { de presión } \\
\text { Anulares }\end{array}$ & $\begin{array}{l}\text { Sistema de cierre } \\
\text { manual }\end{array}$ & $\begin{array}{c}\text { Mala alineación } \\
\text { con boca del } \\
\text { pozo }\end{array}$ & Anillos, bridas & & & & \\
\hline Cement problems & $\begin{array}{c}\text { Falla de equipos } \\
\text { y accesorios }\end{array}$ & $\begin{array}{l}\text { Fraguado } \\
\text { prematuro del } \\
\text { cemento }\end{array}$ & $\begin{array}{l}\text { Corrección de } \\
\text { cementación }\end{array}$ & $\begin{array}{l}\text { Taponamiento de la } \\
\text { línea de flujo con } \\
\text { cemento }\end{array}$ & & & & & & \\
\hline Pega de tubería & $\begin{array}{l}\text { Formaciones no } \\
\text { consolidadas }\end{array}$ & $\begin{array}{l}\text { Formaciones } \\
\text { Fracturadas }\end{array}$ & $\begin{array}{l}\text { Inestabilidad de } \\
\text { Lutitas }\end{array}$ & Cemento Blando & $\begin{array}{l}\text { Chatarra en el } \\
\text { hueco }\end{array}$ & $\begin{array}{l}\text { Formación } \\
\text { permeable }\end{array}$ & $\begin{array}{c}\text { Sobre } \\
\text { balance }\end{array}$ & $\begin{array}{l}\text { Geometría } \\
\text { del pozo }\end{array}$ & $\begin{array}{l}\text { Taponamiento } \\
\text { de la sarta }\end{array}$ & \\
\hline Pesca & $\begin{array}{l}\text { Falla en la } \\
\text { tubería }\end{array}$ & Pega de tubería & $\begin{array}{l}\text { Chatarra en el } \\
\text { hueco }\end{array}$ & $\begin{array}{l}\text { Cable de registros } \\
\text { roto }\end{array}$ & & & & & & \\
\hline $\begin{array}{l}\text { Pérdida de } \\
\text { circulación }\end{array}$ & $\begin{array}{c}\text { Alta } \\
\text { permeabilidad y } \\
\text { porosidad }\end{array}$ & $\begin{array}{l}\text { Formaciones } \\
\text { naturalmente } \\
\text { fracturadas }\end{array}$ & $\begin{array}{l}\text { Inadecuado } \\
\text { peso de lodo }\end{array}$ & & & & & & & \\
\hline Arremetidas & $\begin{array}{c}\text { Densidad } \\
\text { insuficiente del } \\
\text { lodo }\end{array}$ & Suabeo & Pistoneo & $\begin{array}{l}\text { Llenado inadecuado } \\
\text { del pozo }\end{array}$ & $\begin{array}{l}\text { Pérdidas de } \\
\text { circulación }\end{array}$ & $\begin{array}{l}\text { Lodo cortado } \\
\text { por gas }\end{array}$ & & & & \\
\hline
\end{tabular}

Recepción: 11 de enero de 2018

Aceptación: 21 de mayo de 2018 